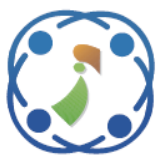

\title{
Performance Evaluation of Face Recognition Based on the Fusion of Bit-Plane and Binary Image Compression Techniques Using Euclidean Distance Classifier
}

\author{
Sunil Swamilingappa Harakannanavar ${ }^{1 *}$ \\ Kori Basava Raja ${ }^{3}$ \\ ${ }^{I} S$ G Balekundri Institute of Technology, Belagavi, Karnataka, India \\ ${ }^{2}$ Dr. Ambedkar Institute of Technology, Bangalore, Karnataka, India \\ ${ }^{3}$ University Visvesvaraya College of Engineering, Bangalore, Karnataka, India \\ * Corresponding author's Email: sunilsh143@gmail.com
}

Prashanth Chikkanayakanahalli Renukamurthy ${ }^{2}$

\begin{abstract}
Personal identification based on face recognition is receiving extensive attention over the last few years in both research and real-time applications due to increasing emphasis on security. In this paper, Face Recognition using the Fusion of bit-plane and binary image compression techniques is presented. Face images are resized to 256 $\times 256$ to obtain the uniformity in the size of face images. The Bit-Plane compression algorithm is applied on the resized image to extract the features of face. Similarly, the Binary image compression technique is applied on $256 \times$ 256 resized images to extract the features. The features produced from both the compression techniques are added to obtain the final facial features. Finally, the test features and database features are compared using Euclidean Distance classifier. Extensive experiments are conducted on combined, Indian female, Indian male and JAFFE face database. The true success rate of the proposed model provides better recognition rate than other existing state-of-the-art methods.
\end{abstract}

Keywords: Face identification, Bit-plane compression, Binary image, Euclidean distance, Success rate.

\section{Introduction}

The main goal of biometric system is to design a system that can provide maximum accuracy and prevent the forgery operation $[4,6]$. There are two phases in biometric systems. First stage is identification phase, which determines the person's identity. Next stage is verification stage, which helps to verify a person's identity in order to distinguish the genuine and imposter candidate $[1,3$, 7]. A face biometric trait is more secured and superior than other traits such as fingerprint, signature scan, iris, hand geometry etc. [1, 5, 6].

In this paper, Face images are resized to $256 \times$ 256 dimensions to meet uniformity throughout the experiments. The bit-plane and binary image compression features are fused to form final features. The features of the test image and database image are compared using Euclidean Distance classifier to decide whether the test image is genuine or imposter. The remaining sections of this paper is organized are as follows. Section 2 discusses the related work of existing techniques. Section 3 describes the proposed face recognition model. The proposed algorithm is given in section 4. The performance analysis is discussed in section 5. Conclusion is given in Section 6.

\section{Related work}

Rangaswamy et al., [1] described overlap local binary pattern to extract the facial features from Discrete Wavelet Transform and Dual Tree Complex Wavelet Transform (DTCWT) for set 1 and set 2 respectively. The features obtained by set 1 and set 2 are concatenated to produce final features. Sagar et al., [2] introduced 2-D Discrete Wavelet Transform (DWT) to produce unique features. The pre-processed images are applied on DWT to 
produce LL features that are normalized to scale the magnitudes ranges from 0 to 1 . Priya et al., [3] presented dual functioned mean based weight matrix to extract the local facial features. Pillai et al., [4] introduced local diagonal extrema number pattern descriptor produces a compact code of face features produced by encoding the detailed information of face images. Now, the micro-patterns are created to extract the directional information. Ramesha et al., [5] described facial algorithm based on gender and age classification. The geometric features of face such as eyes, nose, lips and mouth are located by canny edge detector to produce the edges of face. Sateeshkumar et al., [6] applied Single Tree Wavelet Transform (STWT) on pre-processed images to obtain four sub-bands and extract the facial features. Then, DTCWT approach is applied on face images to obtain the final features. Sagar et al., [7] applied Stationary Wavelet Transform (SWT) on both frontal and non-frontal reference face images of a person to obtain row vectors. Procrustes Analysis (PA) is used to convert the non-frontal to frontal face images. The SWT row vectors of both frontal and non-frontal reference images are concatenated to produce the final features.

Kumar et al., [8] explained average wavelet packet coefficients and Radial Basis Function (RBF) neural network to recognize face. The RBF neural network is used to recognize the average wavelet packet coefficients. Raju et al., [9] explained the fusion of Principal Component Analysis (PCA), Independent Component Analysis (ICA) and DWT descriptors to recognize face. The PCA and ICA are applied on face images to extract the global features of face. The DWT is employed on ICA to produce final features. Taqdir et al., [10] explained the fusion of DWT and LDA using SIFT key to recognize face. The DWT is applied on resized face images to obtain low frequency LL co-efficients to extract the facial features. Kurban et al., [12] explained VGG face deep learning model and energy imaging approach to extract the local facial features and gesture features respectively. Zhihan Xie et al., [13] combined local binary pattern (LBP) and histogram of gradients (HOG) descriptors to extract the texture features of an infrared face and to extract edge features respectively. Xia et al., [14] described the two layer face model to represent the data with training samples and recognize new faces by calculating residuals. Majumder et al., [15] explained the features generated by geometric and appearance approaches are concatenated to obtain the final features. Chakraborty et al., [16] explained local gradient hexa-pattern descriptor to extract the features. Shyam and Singh [17] presented
Augmented Local Binary Pattern (ALBP) to work on both regular and non-regular patterns. The preprocessing process is performed to convert the irregular patterns to regular patterns and these patterns are finally fused with neighbouring regular pattern to extract the discriminatory information using local descriptors. Sable et al., [18] introduced entropy-based volume SIFT based face model after plastic surgery to extract the structural and statistical features. Srivastava et al., [19] proposed TROIKA which enables deep hybrid computational model neural classifier to provide the superior in its computational power, learning and generalization of face. Deshpande et al., [20] combined CDF 5/3 Lift DWT and FFT to recognize the face. The Lifting DWT descriptor is applied on the pre-processed images to extract approximate LL sub-band features and FFT is used to extract the magnitude features. Finally, both the obtained features are fused to obtain desired features.

From the related work of existing face recognition models $[1,2,6,7,9-11,20,21]$, it is observed that the simple pixel level and average level approaches directly affect on the contrast of the image due to blurring effect. The spatial domain approach $[8,10]$ does not have a fixed set of basis vectors hence, the spatial domain fusion produces spectral degradation which affects accuracy of the face model. The final fused images obtained by the transform domain results less spatial resolution which decreases the overall recognition rates. In addition to this the existing models are demonstrated on limited databases for evaluating the performances of existing models.

So in order to overcome the limitations faced by the existing conventional methods, the multi level fusion is performed using bit-plane and binary image compression techniques and provides improved results where the output images includes high spatial resolution with high quality spectral content. The experimental setup is carried on various publically available face databases such as Combined, Indian male, Indian female and JAFFE databases and results superior recognition rates when compared with the different state-of-the-art methods.

\section{Proposed model}

In this section, the face recognition model based on fusion of Bit Plane and Binary image compression techniques are discussed. The proposed model is shown in Fig. 1. Face images are resized to $256 \times 256$ to obtain the uniformity in the size of face 


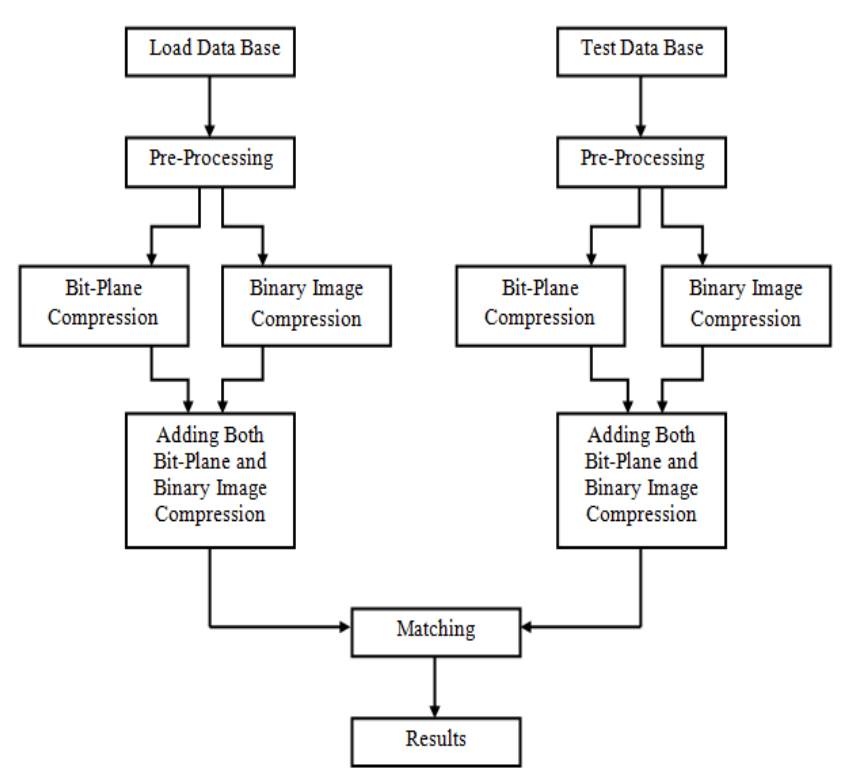

Figure.1 Proposed model

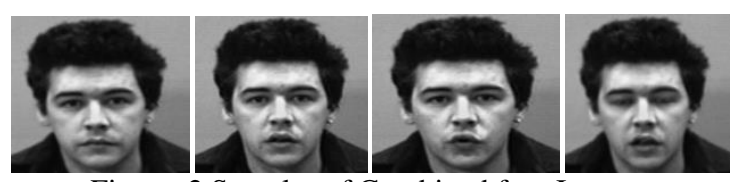

Figure. 2 Samples of Combined face Images

images. The bit-plane compression and binary image compression algorithms are applied on the $256 \times 256$ resized images to extract the facial features. Then the features produced from both the compression techniques are added to obtain the final facial features. Finally, the test features and database features are compared using ED classifier.

\subsection{Face databases}

The publicly available face databases such as Combined, Indian face and JAFFE face database are used to test the proposed model for performance analysis.

\subsubsection{Combined face database (L-speck)}

The database [21] has One hundred and twenty persons with nineteen images per person. Totally the database has two thousand and two hundred and eighty images. The size of each image is $320 \times 280$. The samples of combined face database of one person are shown in Fig. 2.

\subsubsection{Indian female database}

The database [22] has twenty-two persons with eleven images per person. Totally the database has two hundred forty images. The size of each image is $640 \times 480$. The samples of Indian female face database of one person are shown in Fig. 3.

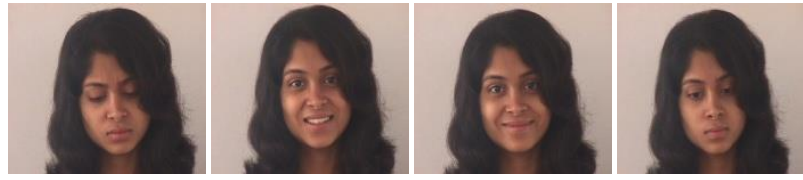

Figure. 3 Samples of Indian female face images

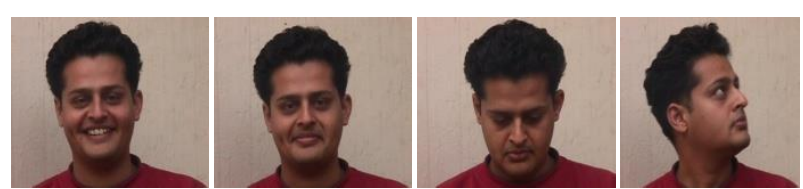

Figure. 4 Samples of Indian male face Images

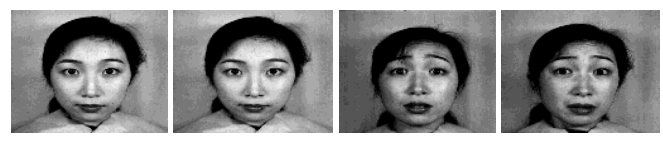

Figure. 5 Samples of JAFFE face images

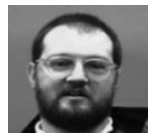

(a)

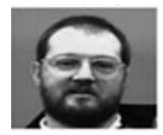

(b)
Figure. 6 Effect of preprocessing: (a) Before preprocessing and (b) Preprocessing output

\subsubsection{Indian Male Database}

The database [22] has twenty persons with eleven images per person. Totally the database has two hundred and twenty images. The size of each image is $640 \times 480$. The samples of Indian male face database of one person are shown in Fig. 4.

\subsubsection{Japanese Female Facial Expression (JAFFE)}

The JAFFE face database [23] has ten persons with twenty images per person. Totally the database contains two hundred images of seven facial expressions. The size of each image is $256 \times 256$. The samples of JAFFE face database of one person are shown in Fig. 5.

\subsection{Preprocessing}

The basic pre-processing effect on combined face database is shown in Fig. 6. The input image from the face databases is cropped and resized to $256 \times 256$ dimensions to meet the uniformity throughout the experiments.

\subsection{Bit-plane compression}

The process of compression is applied to digital images which helps to reduce their cost for storage or transmission. Let us consider a digital image consisting of $m \times n$ pixels with $\mathrm{M}$ rows and $\mathrm{N}$ columns of pixels as shown in equation 1. Here, each pixel is represented by 8-bit gray level value ranging between 0 to 255. Bit planes can be formed with the consideration of eight groups of data in 
accordance to the order of the bits in the image. The least significant bits (LSB) are used to form bitplane 0 whereas bit-plane 7 is formed using the most significant bits (MSB). The process of bitplane extraction is based on decimal to binary conversion for each pixel. The Eq.(2) shows the mathematical derivation for bit-plane ' $i$ ' extraction. For bit-plane ' $i$ ' extraction, the original image elements $f(x, y)$ are rounded to nearest integers which is less than or equal to ' $x$ ' after divisible by $2^{\mathrm{i}}$. The remainder $\mathrm{R}$ is equivalent to information of bitplane $i$.

$$
f(x, y)=\left[\begin{array}{cccc}
f(0,0) & f(0,1) & \ldots & f(0, N-1) \\
f(1,0) & f(1,1) & \ldots & f(1, N-1) \\
\vdots & \vdots & & \vdots \\
f(M-1,0) & f(M-1,1) & \ldots & f(M-1, N-1)
\end{array}\right]
$$

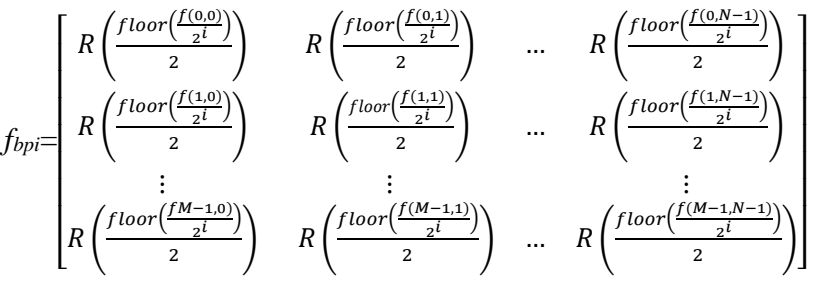

$$
\begin{aligned}
& \approx f_{b p i}(x, y)=R\left[\frac{1}{2} f \operatorname{loor}\left(\frac{1}{2^{i}}[f(x, y)]\right)\right]
\end{aligned}
$$

Fig. 7 which illustrates the bit-planes of a $256 \times 256$ image from combined face database after bit-plane feature extraction performed using equation 2 . It is noticed that the patterns of the face is more visible in the bit plane 7 which has higher order bits contain majority of the visually significant data whereas the remaining lower order bit-planes 0,1,2,3,4,5,6 contains more subtle (more difficult to analyse) details in the image. Because of this reason, the proposed model uses higher order bits for recognition of face.

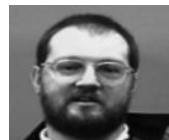

(a)

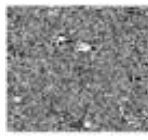

(b)

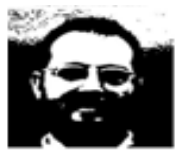

(c)

Figure. 7 Extracted bit-planes features from original image from combined face database: (a) input image, (b) output bit plane (1 bit), and (c) output bit plane ( 8 bit)

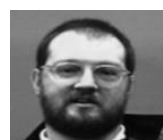

(a)

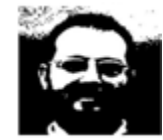

(b)

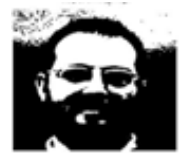

(c)

Figure. 8 Extracted binary features from original image from combined face database: (a) input image, (b) output binary image (1 bit), and (c) output binary image ( 8 bit)

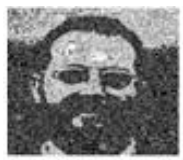

(1 bit)

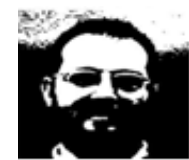

(8 bit)
Figure. 9 Addition of both binary and bit plane

\subsection{Binary image compression}

A binary image contains only two possible values for each pixel. Two colors such as black and white are used to represent the binary images. Binary images are also called 'two-level' which means that each pixel is stored as a single bit either ' 0 ' or ' 1 '. Binary images are often used as masks as a result to perform the operations in digital image processing that includes segmentation, thresholding and dithering. A binary image can be stored in memory as a bitmap. It operates on a $3 \times 3$ window of the image which contains nine pixels i.e., $2^{9}=512$ possible values. By considering only the central pixel, based on the surrounding pixels it is possible to define whether it remains set or unset. It also supports distance transform which gives the distance of every set pixel from the nearest unset pixel. The binary images for the combined face database are given in Fig. 8.

Once the features are generated from the bitplane and binary image compression techniques, it is required to add both the features produced by compression techniques and the results obtained are shown in Fig. 9.

\subsection{Euclidean distance}

The minimum Euclidean Distance gives the similarity between the unknown face image that is being tested and the known ones in the database. In Cartesian coordinates, if $\mathrm{p}$ and $\mathrm{q}$ are two points in Euclidean space, then its distance from $p$ to $q$ is given by equation 3 .

$$
d(p, q)=\sqrt{\left(q_{1}-p_{1}\right)^{2}+\left(q_{2}-p_{2}\right)^{2}+\cdots+\left(q_{n}-p_{n}\right)^{2}}
$$




\section{Proposed algorithm}

\subsection{Problem defination}

The proposed algorithm is given in Table 1 and is tested with various publicly available face databases. The new concept of adding Bit-plane compression image with binary image is introduced to obtain features of face images for better performance. The features of test image and database image are compared using Euclidean Distance classifier.

\subsection{Objectives}

The main objectives of the proposed face model based on the Bit-plane and binary image compression techniques are to

(i) Increase the total success rate and

(ii) Reduce the error rates.

Table1. Proposed Algorithm (Fusion of Bit-Plane and Binary image compression)

Input: Face images from different face databases such as Combined face database, Indian female, Indian female and JAFFE database.

Output: Recognition of face images and Performance Parameters.

Step 1: Face image is read from the facial database.

Step 2: Face Image is resized to $256 \times 256$.

Step 3: Apply bit-plane compression technique on resized image.

Step 4: Apply binary image compression technique on resized image.

Step 5: Addition operation is performed on the output of patterns of bit-plane and binary image Compression techniques.

Step 6: Repeat step 1 to 6 for test image.

Step 7: Test image features are compared with added features using ED classifier.

Step 8: If ED is less than threshold value, then the image is considered as genuine image. Otherwise, it is treated as imposter image.

\section{Performance analysis}

In this section, the definitions of performance parameters, analysis of performance parameters using various face databases and comparison of proposed model with existing methods are discussed.

\subsection{Performance parameters definations}

(i) False Rejection Ration (FRR): The ratio of the number of true persons rejected to the overall persons inside the database as given in Eq. (4).
FRR $=\frac{\text { Number of genuine persons rejected in database }}{\text { Total number of persons inside the database }}$

(ii) False Acceptance Ratio (FAR): The ratio of the number of fake persons accepted as real to the overall persons outside the database as given in Eq. (5).

FAR $=\frac{\text { Number of imposter persons accepted as genuine }}{\text { Total number of persons outside the database }}$

(iii) Total Success Rate (TSR): The ratio of the number of true persons recognized accurately to the overall persons inside the database as given in Eq. (6).

$$
\text { TSR }=\frac{\text { Number of genuine persons recognized correctly }}{\text { Total number of persons inside the database }}
$$

(iv) Equal Error Rate (EER): The EER is the intersection of FRR and FAR as given in Eq. (7).

$$
\mathrm{EER}=\mathrm{FAR}-\mathrm{FRR}
$$

\subsection{Analysis of performance parameters}

The MATLAB software is used for simulation of the proposed model. The simulation is carried out on entire face database for varying persons inside database (PID) and persons outside database (POD). The performance parameters such as FAR, FRR, TSR and EER for different databases such as combined face database, Indian female, Indian male and JAFFE face database are discussed in detail for the proposed model.

\subsubsection{Analysis with combined face database}

The percentage variation of FAR, FRR and TSR with threshold for combined face database is given in shown in Fig 10.

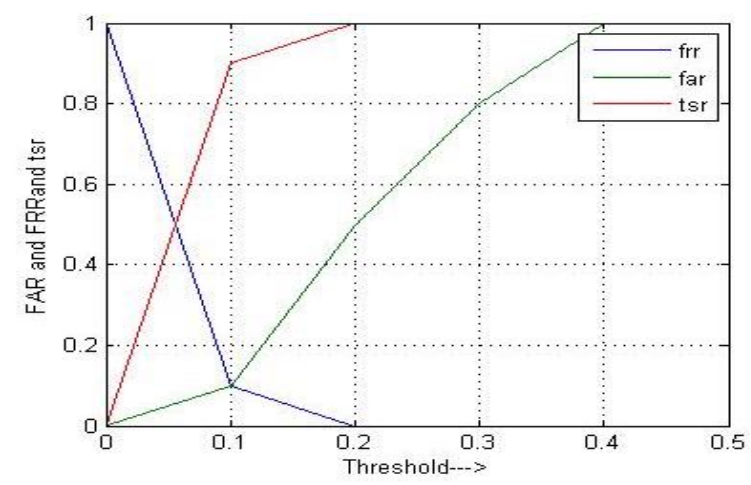

Figure. 10 Plot of FAR, FRR and TSR Versus for PID: $\mathrm{POD}=10: 10$ 


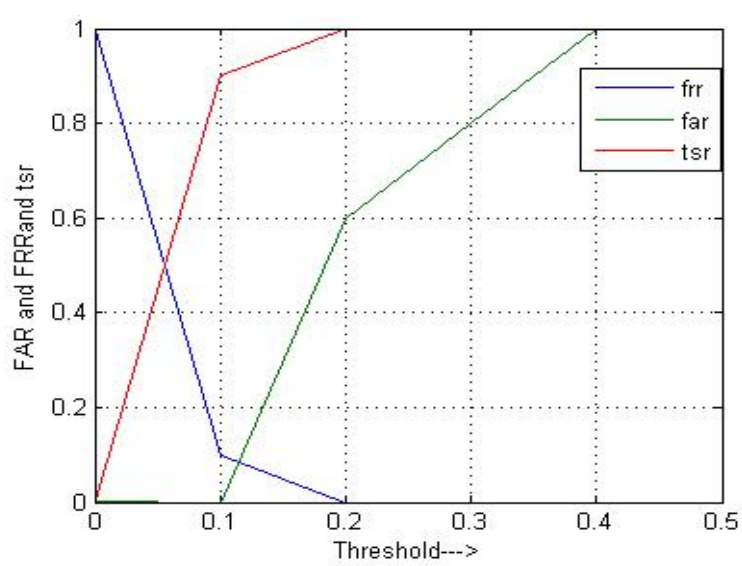

Figure. 11 Plot of FAR, FRR and TSR versus for PID: $P O D=10: 5$

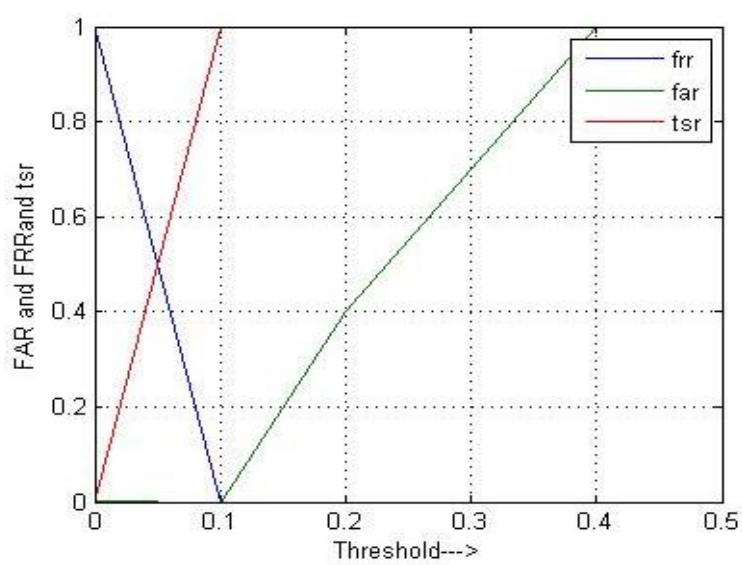

Figure. 12 Plot of FAR, FRR and TSR versus for PID: $\mathrm{POD}=5: 10$

By increasing the value of threshold, the percentage value of FAR and TSR increases. As threshold value decreases, the value of FRR gets reduced from maximum to zero. It is noticed that the maximum TSR for the proposed model is $100 \%$ at a threshold of 0.2. It is observed that the value of EER results $10 \%$ for a threshold of 0.1 .

Fig. 11 shows the percentage variations of FAR and FRR with threshold. As threshold value decreases, the value of FRR gets reduced from maximum to zero. It is noticed that the maximum TSR for the proposed model is $100 \%$ at a threshold of 0.2 and the value of EER results $8 \%$ for a threshold of 0.12 .

Fig. 12 shows the percentage variations of FAR and FRR with threshold. As threshold value decreases, the value of FRR gets reduced from maximum to zero. It is noticed that the maximum TSR for the proposed model is $100 \%$ at a threshold of 0.1 and the value of EER results $0 \%$ for a threshold of 0.1 .

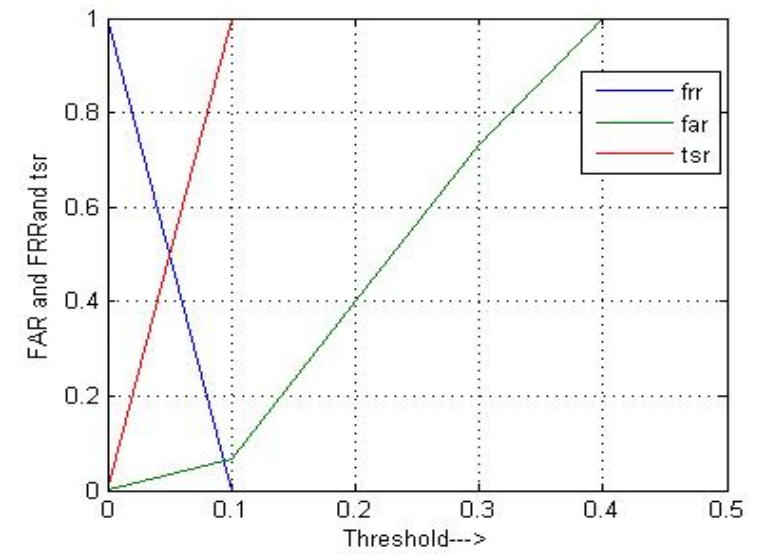

Figure. 13 Plot of FAR, FRR and TSR versus for PID: $\mathrm{POD}=5: 15$

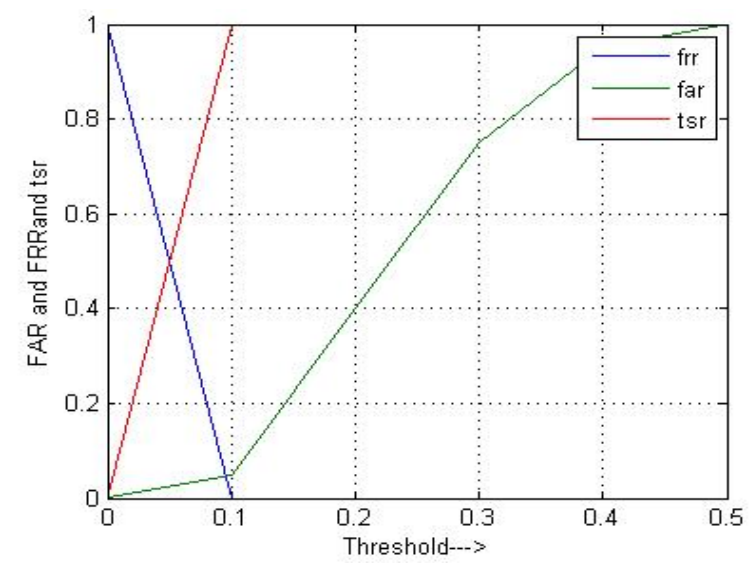

Figure. 14 Plot of FAR, FRR and TSR versus for PID: $\mathrm{POD}=5: 20$

Fig. 13 shows the percentage variations of FAR and FRR with threshold. As threshold value decreases, the value of FRR gets reduced from maximum to zero. It is noticed that the maximum TSR for the proposed model is $100 \%$ at a threshold of 0.1 and the value of EER results $6 \%$ for a threshold of 0.09 .

Fig. 14 shows the percentage variations of FAR and FRR with threshold. As threshold value decreases, the value of FRR gets reduced from maximum to zero. It is noticed that the maximum TSR for the proposed model is $100 \%$ at a threshold of 0.1 and the value of EER results $5 \%$ for a threshold of 0.09 .

Fig. 15 shows the percentage variations of FAR and FRR with threshold. As threshold value decreases, the value of FRR gets reduced from maximum to zero. It is noticed that the maximum TSR for the proposed model is $100 \%$ at a threshold of 0.1 and the value of EER results $4 \%$ for a threshold of 0.09 . 


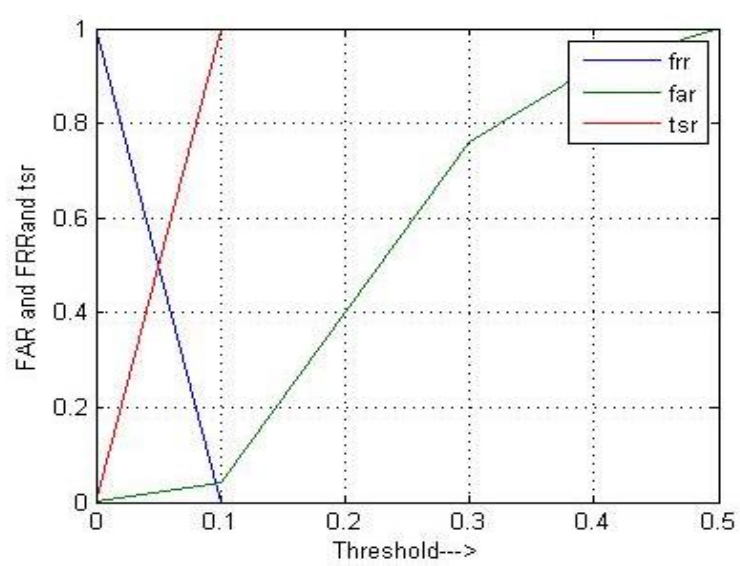

Figure. 15 Plot of FAR, FRR and TSR versus for PID: $\mathrm{POD}=5: 25$

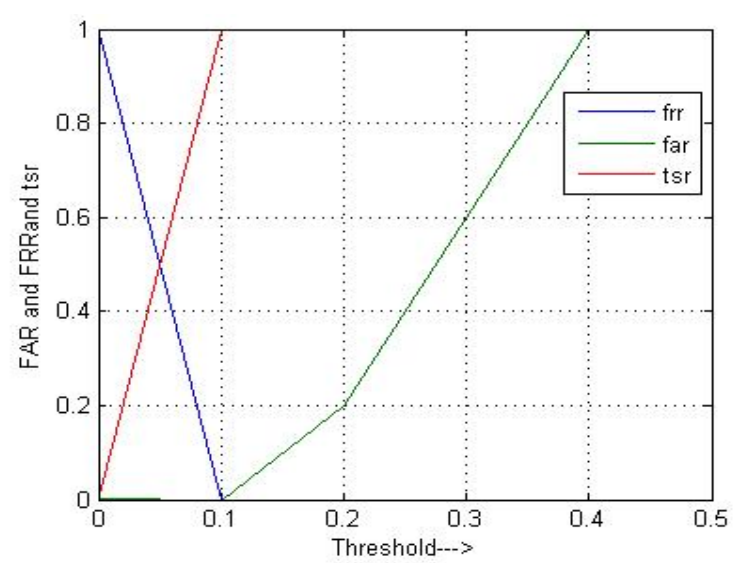

Figure. 16 Plot of FAR, FRR and TSR versus for PID: $\mathrm{POD}=5: 5$

Table 2. Variations of FAR, FRR and TSR for different PID: POD for combined face database

\begin{tabular}{ccccc}
\hline PID & POD & $\begin{array}{c}\text { \% Max. } \\
\text { TSR }\end{array}$ & $\begin{array}{c}\text { \% Opt. } \\
\text { TSR }\end{array}$ & EER \\
\hline 10 & 10 & 100 & 90 & 10 \\
10 & 5 & 100 & 93.3 & 08 \\
5 & 5 & 100 & 100 & 00 \\
5 & 10 & 100 & 100 & 00 \\
5 & 15 & 100 & 97 & 06 \\
5 & 20 & 100 & 98 & 05 \\
5 & 25 & 100 & 99 & 04 \\
\hline
\end{tabular}

Fig. 16 shows the percentage variations of FAR and FRR with threshold. As threshold value decreases, the value of FRR gets reduced from maximum to zero. It is noticed that the maximum TSR for the proposed model is $100 \%$ at a threshold of 0.1 and the value of EER results $0 \%$ for a threshold of 0.1 .

The percentage values of FRR decreases and FAR, TSR increases as varied in threshold value. It is observed that the value of OTSR and max.TSR is
$100 \%$ corresponding to $0 \%$ EER. The variations of percentage EER, OTSR, max. TSR for different variations of PID and POD for combined face database is shown in table 2 and it is recorded that, by increasing POD, the value of OTSR and EER increases and decreases respectively as PID kept constant. Similarly, by increasing PID, the value of OTSR and EER decreases and increases respectively as POD kept constant.

\subsubsection{Analysis with Indian female database}

The percentage variation of FAR, FRR and TSR with threshold for Indian female database is given in Fig. 17.

By increasing the value of threshold, the percentage value of FAR and TSR increases. As threshold value decreases, the value of FRR gets reduced from maximum to zero. It is noticed that the maximum TSR for the proposed model is $30 \%$ at a threshold of 0.1 and the value of EER results $50 \%$ for a threshold of 0.05 .

Fig. 18 shows the percentage variations of FAR and FRR with threshold. As threshold value decreases, the value of FRR gets reduced from maximum to zero. It is noticed that the maximum TSR for the proposed model is $20 \%$ at a threshold of 0.1 and the value of EER results $50 \%$ for a threshold of 0.05 .

Fig. 19 shows the percentage variations of FAR and FRR with threshold. As threshold value decreases, the value of FRR gets reduced from maximum to zero. It is noticed that the maximum TSR for the proposed model is $60 \%$ at a threshold of 0.1 and the value of EER results $50 \%$ for a threshold of 0.066 .

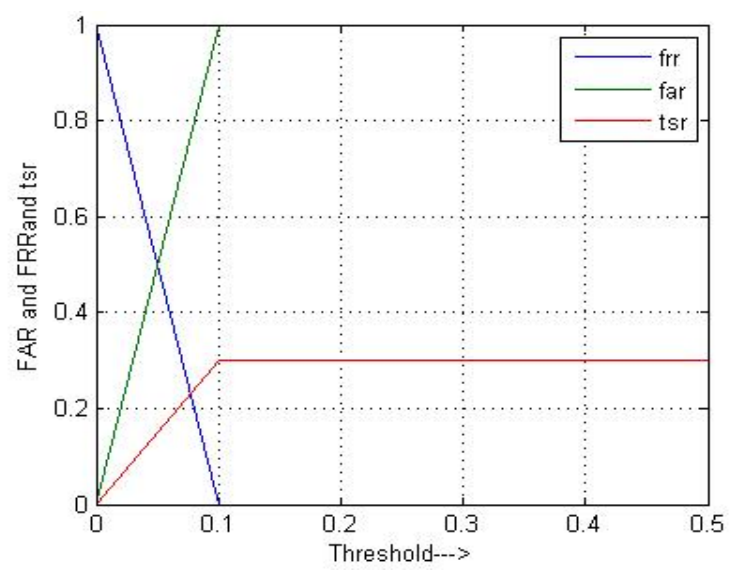

Figure. 17 Plot of FAR, FRR and TSR versus for PID: $\mathrm{POD}=10: 5$ 


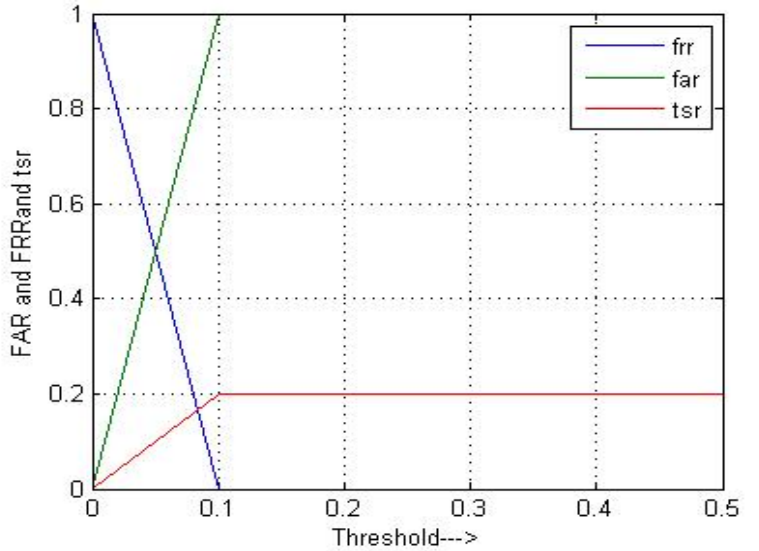

Figure. 18 Plot of FAR, FRR and TSR versus for PID: $\mathrm{POD}=15: 5$

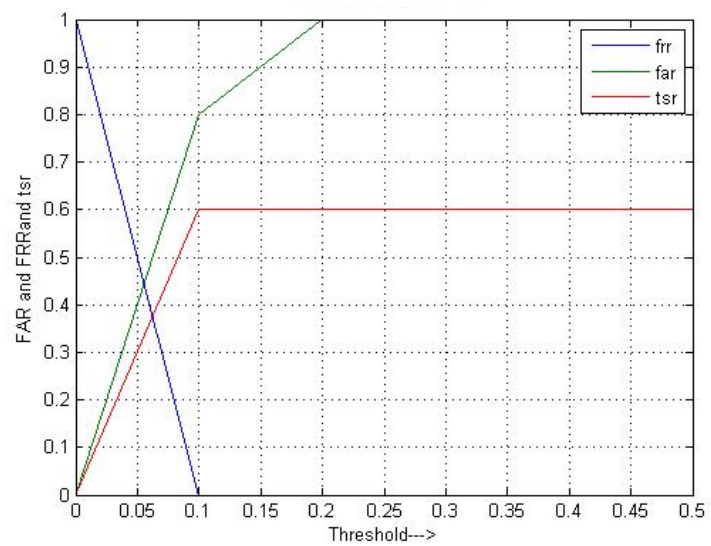

Figure. 19 Plot of FAR, FRR and TSR versus for PID: $\mathrm{POD}=5: 10$

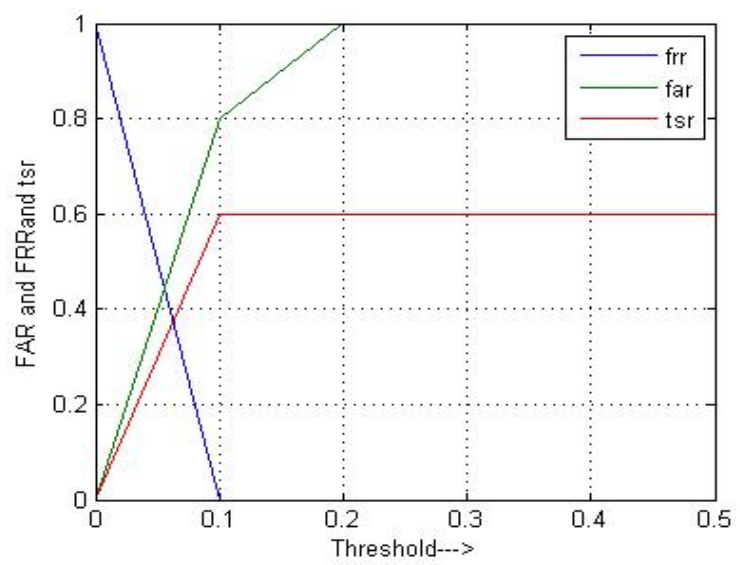

Figure. 20 Plot of FAR, FRR and TSR versus for PID: $\mathrm{POD}=5: 15$

Fig. 20 shows the percentage variations of FAR and FRR with threshold. As threshold value decreases, the value of FRR gets reduced from maximum to zero. It is noticed that the maximum TSR for the proposed model is $60 \%$ at a threshold of 0.1 and the value of EER results $44 \%$ for a threshold of 0.06 .

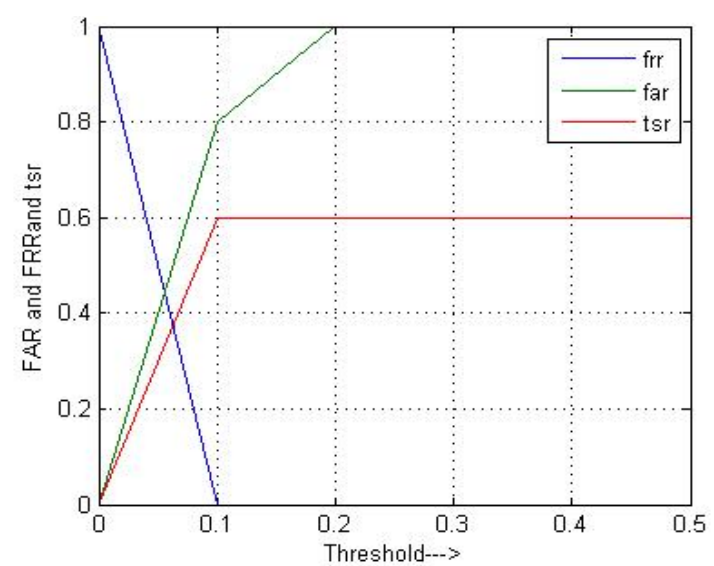

Figure. 21 Plot of FAR, FRR and TSR versus for PID: $\mathrm{POD}=5: 5$

Table 3. Variations of FAR, FRR and TSR for different PID: POD for Indian female database.

\begin{tabular}{ccccc}
\hline PID & POD & $\begin{array}{c}\text { \% Max. } \\
\text { TSR }\end{array}$ & $\begin{array}{c}\text { \% Opt. } \\
\text { TSR }\end{array}$ & EER \\
\hline 15 & 5 & 20 & 10 & 50 \\
10 & 5 & 30 & 15 & 50 \\
5 & 5 & 60 & 35 & 44 \\
5 & 10 & 60 & 33.3 & 50 \\
5 & 15 & 60 & 35 & 44 \\
\hline
\end{tabular}

Fig. 21 shows the percentage variations of FAR and FRR with threshold. As threshold value decreases, the value of FRR gets reduced from maximum to zero. It is noticed that the maximum TSR for the proposed model is $60 \%$ at a threshold of 0.1 and the value of EER results $44 \%$ for a threshold of 0.06 .

The percentage values of FRR decreases and FAR, TSR increases as varied in threshold value. It is observed that the value of OTSR and max.TSR are $35 \%$ and $60 \%$ respectively corresponding to $44 \%$ EER. The variations of percentage EER, OTSR, max. TSR for different variations of PID and POD for Indian female face database is shown in table 3 and it is recorded that, by increasing POD, the value of OTSR and EER increases and decreases respectively as PID kept constant. Similarly, by increasing PID, the value of OTSR and EER decreases and increases respectively as POD kept constant.

\subsubsection{Analysis with Indian male database}

The percentage variation of FAR, FRR and TSR with threshold for Indian male database is given in Fig. 22. 


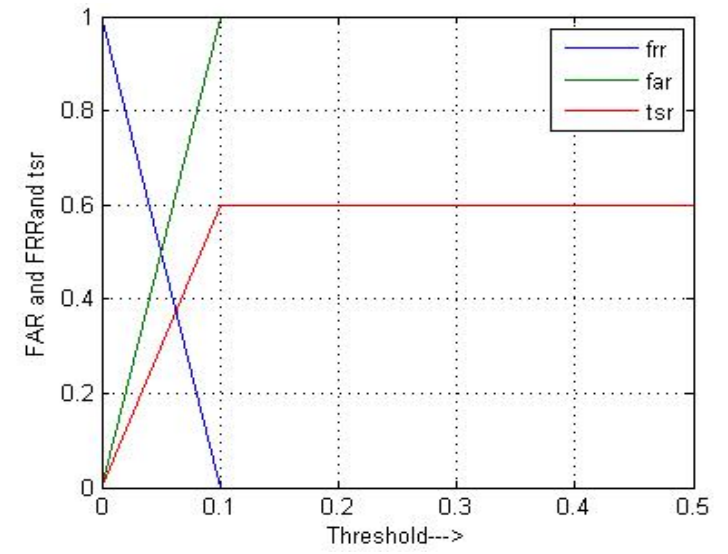

Figure. 22 Plot of FAR, FRR and TSR versus for PID: $\mathrm{POD}=10: 5$

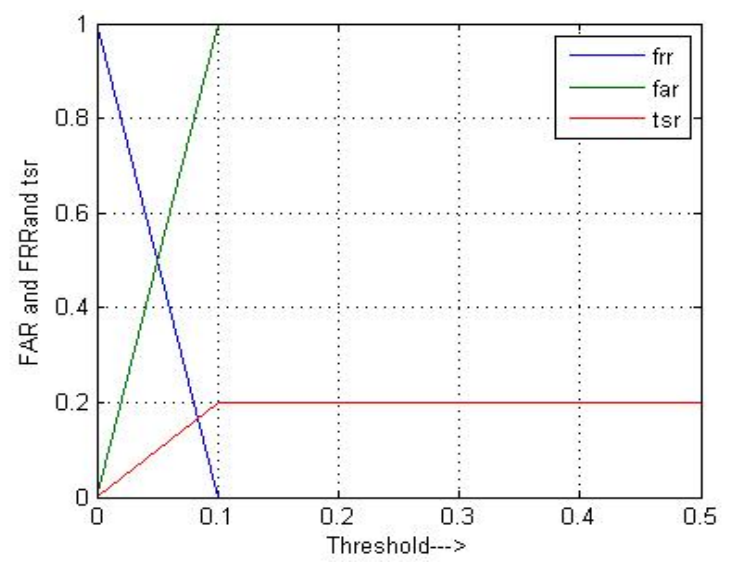

Figure. 23 Plot of FAR, FRR and TSR versus for PID: $\mathrm{POD}=15: 5$

By increasing the value of threshold, the percentage value of FAR and TSR increases. As threshold value decreases, the value of FRR gets reduced from maximum to zero. It is noticed that the maximum TSR for the proposed model is $60 \%$ at a threshold of 0.1 and the value of EER results $50 \%$ for a threshold of 0.05 .

Fig. 23 shows the percentage variations of FAR and FRR with threshold. As threshold value decreases, the value of FRR gets reduced from maximum to zero. It is noticed that the maximum TSR for the proposed model is $20 \%$ at a threshold of 0.1 and the value of EER results $50 \%$ for a threshold of 0.05 .

Fig. 24 shows the percentage variations of FAR and FRR with threshold. As threshold value decreases, the value of FRR gets reduced from maximum to zero. It is noticed that the maximum TSR for the proposed model is $80 \%$ at a threshold of 0.1 and the value of EER results $50 \%$ for a threshold of 0.05 .

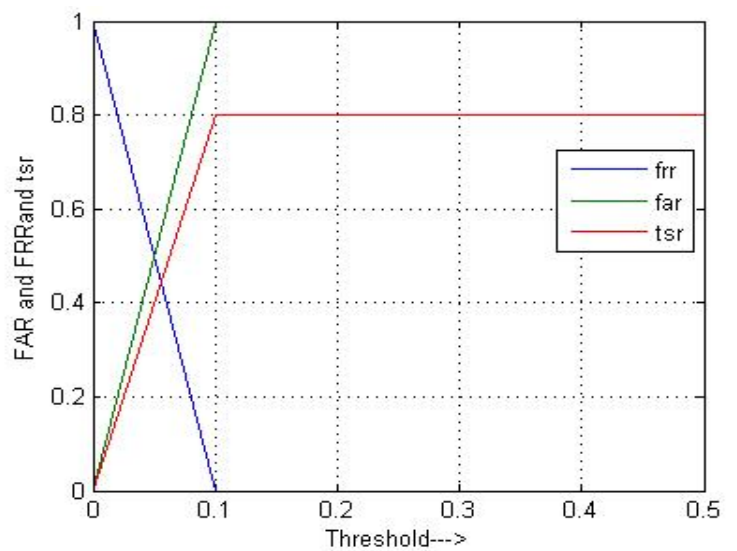

Figure. 24 Plot of FAR, FRR and TSR versus for PID: $\mathrm{POD}=5: 10$

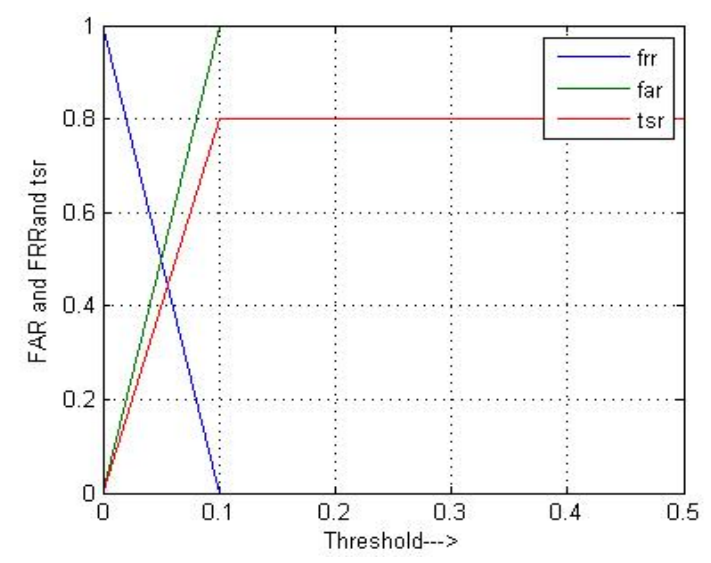

Figure. 25 Plot of FAR, FRR and TSR versus for PID: $\mathrm{POD}=5: 15$

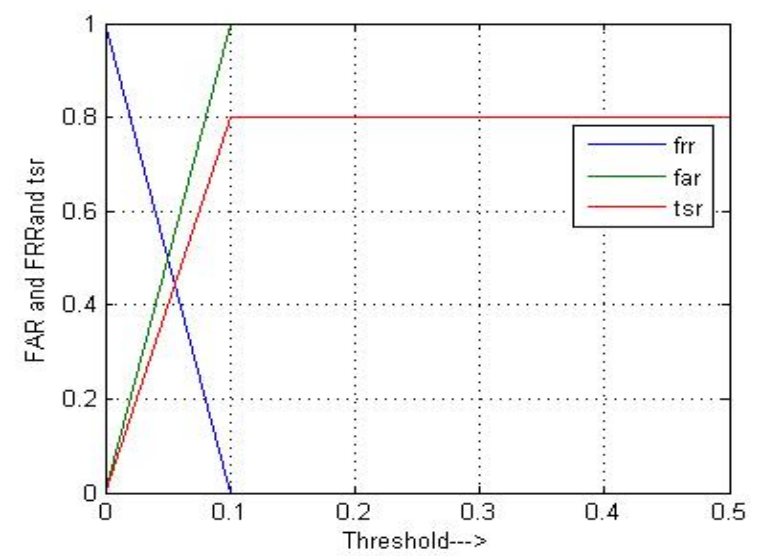

Figure. 26 Plot of FAR, FRR and TSR versus for PID: $\mathrm{POD}=5: 5$

Fig. 25 shows the percentage variations of FAR and FRR with threshold. As threshold value decreases, the value of FRR gets reduced from maximum to zero. It is noticed that the maximum TSR for the proposed model is $80 \%$ at a threshold of 0.1 and the value of EER results $50 \%$ for a threshold of 0.05 . 
Table 4. Variations of FAR, FRR and TSR for different PID: POD for Indian male database

\begin{tabular}{ccccc}
\hline PID & POD & $\begin{array}{c}\text { \% Max. } \\
\text { TSR }\end{array}$ & $\begin{array}{c}\text { \% Opt. } \\
\text { TSR }\end{array}$ & EER \\
\hline 15 & 5 & 20 & 10 & 50 \\
10 & 5 & 60 & 30 & 50 \\
5 & 5 & 80 & 40 & 50 \\
5 & 10 & 80 & 40 & 50 \\
5 & 15 & 80 & 40 & 50 \\
\hline
\end{tabular}

Fig. 26 shows the percentage variations of FAR and FRR with threshold. As threshold value decreases, the value of FRR gets reduced from maximum to zero. It is noticed that the maximum TSR for the proposed model is $80 \%$ at a threshold of 0.1 and the value of EER results $50 \%$ for a threshold of 0.05 .

The percentage values of FRR decreases and FAR, TSR increases as varied in threshold value. It is observed that the value of OTSR and max.TSR are $40 \%$ and $80 \%$ respectively corresponding to $50 \%$ EER. The variations of percentage EER, OTSR, max. TSR for different variations of PID and POD for Indian male face database is shown in table 4 and it is recorded that, by increasing POD, the value of OTSR and EER increases and decreases respectively as PID kept constant. Similarly, by increasing PID, the value of OTSR and EER decreases and increases respectively as POD kept constant.

\subsubsection{Analysis with JAFFE database}

The percentage variation of FAR, FRR and TSR with threshold for JAFFE database is given in Fig. 27. By increasing the value of threshold, the percentage value of FAR and TSR increases. As threshold value decreases, the value of FRR gets reduced from maximum to zero. It is noticed that the maximum TSR for the proposed model is $100 \%$ at a threshold of 0.1 and the value of EER results $0 \%$ for a threshold of 0.1 .

Fig. 28 shows the percentage variations of FAR and FRR with threshold. As threshold value decreases, the value of FRR gets reduced from maximum to zero. It is noticed that the maximum TSR for the proposed model is $82.3 \%$ at a threshold of 0.2 and the value of EER results $50 \%$ for a threshold of 0.1 .

Fig. 29 shows the percentage variations of FAR and FRR with threshold. As threshold value decreases, the value of FRR gets reduced from maximum to zero. It is noticed that the maximum

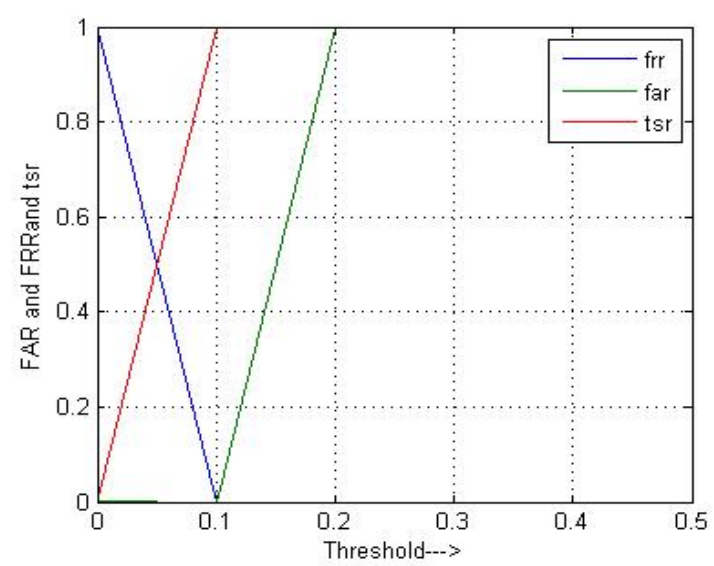

Figure. 27 Plot of FAR, FRR and TSR versus for PID: $\mathrm{POD}=2: 2$

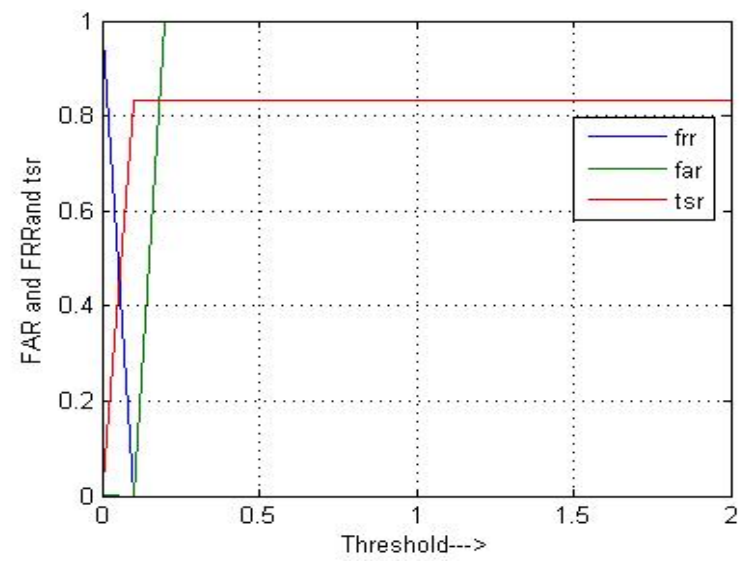

Figure. 28 Plot of FAR, FRR and TSR versus for PID: $\mathrm{POD}=6: 2$

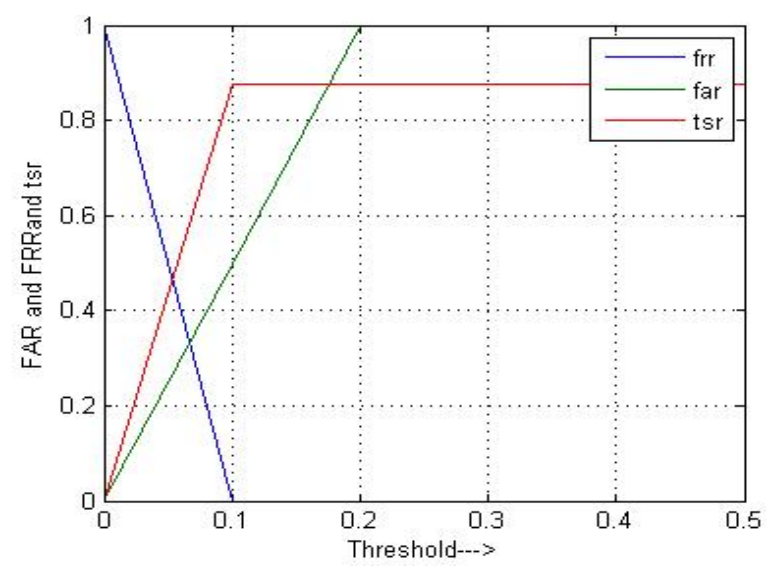

Figure. 29 Plot of FAR, FRR and TSR versus for PID: $\mathrm{POD}=8: 2$

TSR for the proposed model is $87.5 \%$ at a threshold of 0.1 and the value of EER results $50 \%$ for a threshold of 0.1 .

The percentage values of FRR decreases and FAR, TSR increases as varied in threshold value. It is observed that the value of OTSR and max.TSR is $100 \%$ corresponding to $0 \%$ EER. The variations of percentage EER, OTSR, max. TSR for different 
Table 5. Variations of FAR, FRR and TSR for different PID: POD for JAFFE face database

\begin{tabular}{ccccc}
\hline PID & POD & $\begin{array}{c}\text { \% Max. } \\
\text { TSR }\end{array}$ & $\begin{array}{c}\text { \% Opt. } \\
\text { TSR }\end{array}$ & EER \\
\hline 8 & 2 & 87.5 & 58 & 33.3 \\
2 & 2 & 100 & 100 & 0 \\
6 & 2 & 82.3 & 82.3 & 0 \\
\hline
\end{tabular}

Table 6. Comparison of TSR value of the proposed system with existing system on combined face database

\begin{tabular}{llc}
\multicolumn{1}{c}{ Authors } & \multicolumn{1}{c}{ Techniques } & $\begin{array}{l}\text { Max. } \\
\text { TSR } \\
(\boldsymbol{\%})\end{array}$ \\
\hline $\begin{array}{l}\text { Sateeshkumar H C } \\
\text { et al., [6] }\end{array}$ & $\begin{array}{l}\text { STWT and DTCWT } \\
\text { using 2D Q-shift } \\
\text { filters }\end{array}$ & 99.15 \\
$\begin{array}{l}\text { G. V. Sagar et al., } \\
\text { [7] }\end{array}$ & SWT and PA \\
$\begin{array}{l}\text { Erum Naz et al., } \\
\text { [24] }\end{array}$ & PCA and FDA \\
$\begin{array}{l}\text { Ramesha et al., } \\
\text { [25] }\end{array}$ & $\begin{array}{l}\text { DTCWT } \\
\text { Proposed Model }\end{array}$ & $\begin{array}{l}\text { Fusion of Bit-Plane } \\
\text { and Binary image } \\
\text { compression. }\end{array}$ \\
\hline
\end{tabular}

Table 7. Comparison of TSR value of the proposed system with existing system on JAFFE face database

\begin{tabular}{llc}
\hline \multicolumn{1}{c}{ Authors } & \multicolumn{1}{c}{ Techniques } & $\begin{array}{c}\text { Max. } \\
\text { TSR } \\
(\mathbf{\%})\end{array}$ \\
\hline $\begin{array}{l}\text { Y. P. Chen et al., } \\
{[26]}\end{array}$ & $\begin{array}{l}\text { Extended Local Binary } \\
\text { Pattern }\end{array}$ & 95 \\
$\begin{array}{l}\text { S. Kumar et al., } \\
\text { [27] }\end{array}$ & $\begin{array}{l}\text { Weighted Projection } \\
\text { based Local Binary } \\
\text { Pattern } \\
\text { Fusion of Bit-Plane } \\
\text { Proposed Model Binary image } \\
\text { compression }\end{array}$ & $\mathbf{1 0 0}$ \\
\hline
\end{tabular}

variations of PID and POD for JAFFE face database is shown in table 5 and it is recorded that, by increasing POD, the value of OTSR and EER increases and decreases respectively as PID kept constant. Similarly, by increasing PID, the value of OTSR and EER decreases and increases respectively as POD kept constant.

The percentage of maximum TSR of the proposed model is compared with existing methods presented by Sateeshkumar H C et al., [6] adopted STWT and DTCWT descriptors to extract the facial features. G. V. Sagar et al., [7] adopted SWT and PA to extract the facial features for face recognition. Erum Naz et al., [24] adopted PCA and FDA descriptors to extract the facial features and Ramesha et al., [25] have used DTCWT descriptor to generate the facial features for face recognition. It is found that the performance of the proposed method based on the fusion of Bit Plane and Binary image compression techniques is better than the existing methods on combined face database given in Table 6.

The percentage of maximum TSR of the proposed model is compared with existing methods presented by Y. P. Chen et al., [26] adopted Extended Local Binary Pattern (which describes the relationship between pixel and its neighbors with radius ' $R$ ') and Principal Component analysis descriptors to extract the facial features. The obtained features are then classified using Sparse representation classifier. S. Kumar et al., [27] have used Local Binary Pattern descriptor to extract discriminative features from informative regions of face and the extracted informative region will estimates the importance of sub regions using projection analysis of expressive images. It is found that the performance of the proposed method is better than the existing methods on JAFFE face database given in Table 7.

\section{Conclusion}

In this paper, Face Recognition using fusion of Bit-Plane and Binary image compression techniques are presented. Face images are cropped and resized to $256 \times 256$ dimensions. The Bit Plane and Binary image compression algorithms are applied on resized image to extract the features of face. Then the features produced from both the compression techniques are added to obtain the final facial features. Finally, the test features and database features are compared using Euclidean Distance.

The FAR, FRR and TSR versus Threshold for various databases such as combined, Indian male, Indian female and JAFFE database for varying PID:POD are plotted. The true success rate of the proposed model for combined face database and JAFFE shows superior recognition rate of $100 \%$ due to the fusion of Bit-Plane and Binary image compression technique. The proposed face model can be improved by considering the fusion of transform domain and spatial domain features.

\section{References}

[1] Y. Rangaswamy, K. B. Raja, K.R. Venugopal, and L. M. Patnaik, "An OLBP based Transform Domain Face Recognition", International Journal of Advanced Research in Electrical, Electronics and Instrumentation Engineering, Vol. 3, No. 1, pp. 6851-6868, 2014. 
[2] G. V. Sagar, S. Y. Barker, K. B. Raja, K. S. Babu, and K. R. Venugopal, "Convolution based Face Recognition using DWT and Feature Vector Compression", In: Proc. of IEEE International Conference on Image Information Processing, pp. 444-449, 2015.

[3] G. N. Priya and R. S. D. W. Banu, "Occlusion invariant face recognition using mean based weight matrix and support vector machine", International Journal of Indian Academy of Science, Vol. 39, No. 2, pp. 303-315, 2014.

[4] A. Pillai, R. Soundrapandiyan, S. Satapathy, S. Satapathy, K. Jung, and R. Krishnan, "Local diagonal extrema number pattern: A new feature descriptor for face recognition", International Journal of Future Generation Computer Systems, Vol. 81, pp. 297-306, 2018.

[5] K. Ramesha, K. B. Raja, K.R. Venugopal, and L. M. Patnaik, "Feature Extraction based Face Recognition, Gender and Age Classification", International Journal on Computer Science and Engineering, Vol. 2, No. 1, pp. 14-23, 2010.

[6] H.C. Sateeshkumar, C. C. Reddy, K. B. Raja, and K.R. Venugopal, "Face Recognition based on STWT and DTCWT using two dimensional Q-shift Filters", International Journal of Engineering and Research, Vol. 7, No. 1, pp. 64-79, 2017.

[7] G. V. Sagar, S. Reddy, K. S. Babu, K. B. Raja, and K.R. Venugopal, "Face Recognition based on SWT and Procrustes Analysis", International Journal of Computer Science, Vol. 5, No. 9, pp. 57-74, 2017.

[8] T. Kathirvalavakumar and J. B. Vasanthi, "Face Recognition based on Wavelet Packet Coefficients and Radial Basis Function Neural Networks", International Journal of Intelligent Learning Systems and Applications, Vol. 5, No. 2, pp. 115-122, 2013.

[9] K. Raju and Y. S. Rao, "Face Recognition using 2-DPCA, ICA, 2-DWT, Neural Network and SVM", International Journal of Control Theory and Applications, Vol. 10, No. 35, pp. 49-64, 2017.

[10] A. Taqdir and R. Dhir, "Face Recognition using SIFT Key with Optimal Features Selection Model", International Journal of Advance Computer Science and Applications, Vol. 8, No. 2, pp. 403-409, 2017.

[11] S. Ganesan and M. R. Mohammed, "A hybrid face image contrast enhancement technique for improved Face Recognition Accuracy", International Journal of Intelligent Engineering and Systems, Vol. 10, No. 6, pp. 106-115, 2017.
[12] O. C. Kurban, T. Yildirim, and A. Bilgic, "A Multi-Biometric Recognition System based on Deep Features of Face and Gesture Energy Image", In: Proc. of IEEE International Conference on Innovations in Intelligent Systems and Applications, pp. 361-364, 2017.

[13] Z. Xie, P. Jiang, and S. Zhang, "Fusion of LBP and HOG using Multiple Kernel Learning for Infrared Face Recognition", In: Proc. of IEEE International Conference on Computer and Information Science, pp. 81-84, 2017.

[14] Z. Xia, X. Peng, X. Feng, and A. Hadid, "Scarce face recognition via two-layer collaborative representation", International Journal of IET Biometrics, Vol. 7, No. 1, pp. 5662, 2018.

[15]A. Majumder, L. Behera, and V. K. Subramanian, "Automatic Facial Expression Recognition system using Deep Network-based Data Fusion", IEEE Transactions on Cybernetics, Vol. 48, No. 1, pp. 103-114, 2018.

[16] S. Chakraborty, S. Singh, and P. Chakraborty, "Local Gradient Hexa Pattern: A Descriptor for Face Recognition and Retrieva", IEEE Transactions on Circuits and Systems for Video Technology, Vol. 28, No. 1, pp. 171-180, 2018.

[17] R. Shyam and Y. N. Singh, "Face Recognition using uncontrolled environment", International Journal of Advanced Computer Science and Applications, Vol. 7, No. 8, pp. 327-333, 2016.

[18] A. H. Sable, S. N. Talbar, and H. Dhirbasi, "Recognition of plastic surgery faces and the surgery types: An approach with entropy based scale invariant features", International Journal of Computer and Information Sciences, Vol. 10, No. 6, pp. 1-7, 2017.

[19] S. Srivastava and B. Tripathi, "Deep Hybrid Computational Model for Face Recognition", International Journal of Intelligent Engineering and Systems, Vol. 11, No. 1, pp. 121-130, 2018.

[20] N. Deshpande and S. Ravishankar, "Face Recognition Credentials using Fusion of CDF 5/3 Lift Discrete Wavelet Transform and FFT", International Journal of Advances in Computational Science and Technology, Vol. 10, No. 3, pp. 423-443, 2017.

[21] L-spacek database, http://cswww.essex.ac.

[22] Indian face database http://viswww.cs.umass.edu

[23] JAFFE database, http://www.kasrl.org/jaffe.

[24] E. Naz, U. Farooq, and T. Naz, "Analysis of Principal Component Analysis based and fisher discriminant analysis based face recognition algorithms", In: Proc. of IEEE International Conference on Emerging Technologies, pp. 121127, 2006. 
[25] K. Ramesha and K. B. Raja, "Dual Transform based Feature Extraction for face recognition", International Journal of Computer Science, Vol. 8, No. 5, pp. 115-121, 2011.

[26] Y. P. Chen, Q. Chen, K. Y. Chou, and R. Wu "Low cost face recognition system based on Extended Local Binary Pattern", In: Proc. of IEEE International Conference on Automatic Control, pp. 13-18, 2016.

[27] S. Kumar, M. Bhuyan, and B. Chakraborty, "Extraction of Informative regions of a face for facial expression recognition", Research article on Institution of Engineering and Technology, Vol.10, No. 6, pp. 567-576, 2016. 